\title{
Puericultura em Enfermagem: perfil e principais problemas encontrados em crianças menores de um ano
}

\author{
Childcare in Nursing: profile and main problems found in children less than one year \\ Puericultura en Enfermería: perfil y principales problemas encontrados en menores de un año
}

\section{Daiane Porto Gauterio', Denise de Azevedo Irala", Marta Regina Cezar-Vaz"'}
'Secretaria Municipal de Saúde do Rio Grande, Programa de Saúde da Família. Rio Grande-RS, Brasil.
"Hospital Universitário Dr. Miguel Riet Correa Jr. Rio Grande-RS, Brasil.
I" Universidade Federal do Rio Grande, Escola de Enfermagem. Rio Grande-RS, Brasil.

Submissão: 20-06-2011 Aprovação: 31-07-2012

\section{RESUMO}

Estudo descritivo exploratório que teve por objetivos descrever o perfil da população menor de um ano atendida na consulta de enfermagem em Puericultura em uma Unidade Básica de Saúde, em Rio Grande-RS, e avaliar a associação entre situação nutricional e presença de candidíase oral, candidíase perineal e dermatite irritativa das fraldas. A amostra foi constituída por 96 prontuários das crianças menores de um ano que realizaram consulta de enfermagem em puericultura. A maioria das crianças era do sexo feminino e encontrava-se eutrófica; todas as que necessitaram de avaliação pediátrica foram encaminhadas; a dermatite irritativa das fraldas foi a alteração mais frequente e esteve associada à situação nutricional desfavorável; o aleitamento materno foi realizado, no máximo, até o sétimo mês de vida do bebê. O estudo aponta a necessidade de sensibilizar a população a respeito da importância da puericultura para a prevenção de agravos e promoção da saúde das crianças.

Descritores: Saúde da Criança; Enfermagem Pediátrica; Atenção Primária à Saúde.

\begin{abstract}
Descriptive exploratory study that aimed to describe the profile of a population less than one year old of age, assisted in the nursing appointment in Childcare at a Health Basic Unit, in Rio Grande-RS; and to evaluate the association between the nutritional situation and the presence of oral candidiasis, perineal candidiasis and irritative dermatitis due to the diapers. The sample was constituted by all the children's charts (96 overall) who were less than one year old and who were assisted in a nursing appointment in Childcare. Most of the children were girls and were found eutrophic; all the ones who needed a pediatric evaluation were sent to the proper evaluation; the irritative dermatitis of the diapers was the most frequent alteration, and it was associated to the unfavorable nutritional situation; breastfeeding took place, at most, up to the seventh month of the baby. The study indicates the need to sensitize the population to about the importance of childcare for the prevention of injuries and promotion of child health.
\end{abstract}

Key words: Child Health; Pediatric Nursing; Primary Health Care.

\section{RESUMEN}

Estudio descriptivo-exploratorio que tuvo como objetivo describir el perfil de la población con edad menor que un año, atendida en una consulta de enfermería en el Cuidado del Niño en una Unidad Básica de Salud, en Rio Grande (RS), y evaluar la asociación entre el estado nutricional y la presencia de candidiasis bucal, candidiasis perineal y dermatitis causadas por pañales. La muestra se ha constituido por 96 relatos clínicos de los niños menores de un año que realizaron consulta de enfermería en el Cuidado del Niño. La mayoría de los niños eran del sexo femenino y estaban bien nutridos; todos los que necesitaron de evaluación pediátrica fueron encaminados; la dermatitis causada por el uso de pañales fue la alteración más frecuente y estaba asociado con el estado nutricional desfavorable; la lactancia materna fue realizada, hasta el séptimo mes de vida del bebé. El estudio pone de relieve la necesidad de sensibilizar la población acerca de la importancia del Cuidado del Niño para la prevención de enfermedades y promoción de la salud de los niños.

Palabras clave: Salud del Niño; Enfermería Pediátrica; Atención Primaria de Salud. 


\section{INTRODUÇÃO}

A desnutrição nos primeiros anos de vida, refletida por indicadores antropométricos do estado nutricional, é um dos maiores problemas de saúde enfrentados por países em desenvolvimento. Há evidências exaustivas de que déficits de crescimento na infância estão associados à maior mortalidade, excesso de doenças infecciosas, prejuízo para o desenvolvimento psicomotor, menor aproveitamento escolar e menor capacidade produtiva na idade adulta ${ }^{(1-2)}$. Por essas razões, e por sua íntima relação com a pobreza, uma das Metas para o Desenvolvimento do Milênio, assumida em 2000 pelas Nações Unidas, diz respeito à redução à metade da prevalência de déficits de crescimento em crianças menores de 5 anos $^{(3)}$.

No Brasil, segundo o Ministério da Saúde, o acompanhamento do crescimento e desenvolvimento faz parte da avaliação integral à saúde da criança (0 a 6 anos), sendo parte integrante da puericultura, a qual envolve a avaliação do peso, altura, desenvolvimento neuropsicomotor, vacinação e intercorrências, o estado nutricional, bem como orientações à mãe/família/cuidador sobre os cuidados com a criança (alimentação, higiene, vacinação e estimulação) em todo atendimento, não deixando também de registrar todos os procedimentos no cartão da criança ${ }^{(4)}$.

O acompanhamento do crescimento e do desenvolvimento infantil deve ser feito de forma regular, de modo que seja possível a detecção precoce de alterações, viabilizando as devidas condutas em tempo hábil, com o objetivo de proporcionar à criança oportunidades para um desenvolvimento adequado durante toda a infância, contribuindo para que suas potencialidades sejam desenvolvidas, de forma a refletir positivamente por toda a vida ${ }^{(5)}$. No Brasil, o principal agente responsável por esse acompanhamento nos serviços de atenção primária à saúde tem sido o enfermeiro ${ }^{(6)}$.

O acompanhamento do desenvolvimento deve ser realizado na atenção primária à saúde, e deve contar com o apoio da família, comunidade e profissionais da saúde. Cabe ao enfermeiro deter o conhecimento necessário para avaliação da criança, tomada de decisões e orientação da família. Para oferecer um cuidado integral e humanizado, o enfermeiro necessita considerar a criança no contexto socioeconômico, cultural e familiar no qual está inserida ${ }^{(6)}$.

Dentro da atenção básica, a puericultura surge como ferramenta oportuna no acompanhamento integral do crescimento e desenvolvimento infantil, voltando-se para os aspectos de prevenção, proteção e promoção da saúde, de modo que a criança alcance a vida adulta sem influências desfavoráveis trazidas da infância. Neste nível de assistência, o profissional, ao utilizar a consulta de enfermagem em puericultura, necessita buscar o atendimento integral das necessidades da criança, modificando o enfoque centrado na doença. Por intermédio da consulta é possível monitorar, avaliar e intervir no processo de saúde/doença, revelando forte componente interacional e educativo.

As crianças menores de um ano que são levadas à consulta de enfermagem em puericultura podem apresentar problemas relacionados ao aleitamento materno, à candidíase oral e perineal, à dermatite irritativa das fraldas, entre outros. Eles podem ser manejados de forma simples e ser solucionados através de orientações fornecidas pelo enfermeiro durante a puericultura.

O objetivo deste estudo é descrever o perfil da população menor de um ano atendida na consulta de enfermagem em puericultura em uma Unidade Básica de Saúde, na cidade do Rio Grande/RS e avaliar a associação entre situação nutricional desfavorável e presença de candidíase oral, candidíase perineal e dermatite irritativa das fraldas.

\section{METODOLOGIA}

Trata-se de um estudo descritivo exploratório que utilizou dados secundários do banco de dados do projeto de pesquisa intitulado "Trabalho em Saúde e o Contexto Tecnológico na Política de Atenção à Saúde da Família", realizado no período de 2005 a 2008, do qual foram extraídos os dados correspondentes ao período de 16/07/2007 a 16/07/2008.

O estudo base foi realizado em uma Unidade Básica de Saúde (UBS), localizada em um balneário, em Rio Grande/ RS. A UBS presta atendimento 24 horas em situações eletivas, de urgência e de emergência. Conta com uma equipe de saúde multiprofissional (enfermeiro, técnico e auxiliar de enfermagem, médico pediatra e clínico geral, odontólogo e nutricionista). A população atendida pela UBS é constituída por moradores residentes no balneário e pela população flutuante (turistas que frequentam o balneário durante o verão).

A referida UBS foi escolhida para compor o projeto, pois estava prevista sua inclusão na expansão da Estratégia Saúde da Família e, desse modo, a clientela atendida na UBS seria posteriormente acolhida nessa modalidade de atendimento.

Na UBS a primeira consulta de puericultura é realizada pelo pediatra, e as demais consultas, pelo enfermeiro, que encaminha a criança ao médico quando há alteração no exame físico. A criança tem consultas de enfermagem em puericultura agendadas na frequência de uma vez por mês ou a cada 15 dias, no caso de apresentar risco nutricional. Os dados do estudo foram coletados dos prontuários das crianças menores de um ano, que realizaram consulta de enfermagem em puericultura na UBS, no período de 16 de abril de 2007 a 16 de abril de 2008. Ao todo, foram 96 crianças.

Os dados foram transcritos manualmente para um instrumento de coleta, com as seguintes variáveis: sexo, idade em meses, peso, comprimento, encaminhamento para pediatra, aleitamento, número de consultas e situação do acompanhamento, candidíase oral, candidíase perineal e dermatite irritativa das fraldas. As variáveis de peso, altura e idade foram utilizadas para determinar o estado nutricional da criança e a situação nutricional.

Os dados coletados foram introduzidos em um banco desenvolvido no software EPI-INFO, versão 6.04 (Center of Disease Control and Prevention, Atlanta, Estados Unidos), em duas entradas distintas e, posteriormente, inter-relacionados através do comando validate, para verificação de erros.

A análise foi realizada através do programa SPSS 15.0. A análise se compôs de duas etapas: descritiva e bivariada. $\mathrm{Na}$ 
primeira, foi feita uma descrição geral dos dados coletados e, na bivariada, o cruzamento das variáveis dependentes (situação nutricional e número de consultas realizadas) com as variáveis independentes (todas as demais), individualmente. Para calcular a significância estatística das associações, utilizou-se o teste Qui-quadrado $\left(\mathrm{X}^{2}\right)$, tendo sido considerado um nível de significância onde $p \leq 0,05$.

O projeto de pesquisa "Trabalho em Saúde e o Contexto Tecnológico na Política de Atenção à Saúde da Família" foi aprovado pelo Comitê de Ética em Pesquisa na Área da Saúde da Universidade Federal do Rio Grande, sob o número 02/2004.

\section{RESULTADOS}

Das 96 crianças que fizeram parte do estudo, 51 (53\%) eram do sexo feminino. Em relação ao estado nutricional das mesmas, na última consulta realizada, 77 (80,2\%) encontravam-se eutróficas; duas $(2,1 \%)$, em risco nutricional; 15 $(15,7 \%)$, com desnutrição leve; uma $(1,0 \%)$, com desnutrição moderada; e uma (1,0\%), em desnutrição grave (Tabela 1).
Nos casos em que foi realizado encaminhamento do enfermeiro para o pediatra, $65(70,6 \%)$ das crianças foram encaminhadas por alteração no exame físico; 18 (19,6\%) para prescrição de vitamina A e ferro; e nove $(9,8 \%)$ foram encaminhadas a pedido da mãe.

Quanto à variável aleitamento materno exclusivo, verificou-se que a prática se manteve até o sétimo mês de vida de algumas crianças, apresentando percentual maior no terceiro $(38,4 \%)$ e menor no sétimo $(3,1 \%)$.

A porcentagem de crianças que abandonaram o acompanhamento foi de 49,0\% (47). O número de crianças em acompanhamento era de 36 , o que correspondeu a $37,5 \%$. Finalizaram o acompanhamento 13 crianças (13,5\%).

Observou-se que $17(17,7 \%)$ crianças apresentaram candidíase perineal, $10(10,4 \%)$ apresentaram candidíase oral, e 33 $(34,4 \%)$, dermatite irritativa de fraldas.

Não houve associação entre presença de candidíase perineal e oral e situação nutricional desfavorável. Houve, contudo, associação entre presença de dermatite amoniacal e situação nutricional desfavorável $(\mathrm{p}=0,01$ e fischer $=0,02)$ (Tabela 2).

Tabela 1 - Estado nutricional apresentado pelas crianças na última consulta realizada. Rio Grande-RS, 2008.

\begin{tabular}{|c|c|c|}
\hline Estado Nutricional & $(n=96)$ & $\%$ \\
\hline Eutrófico & 77 & 80,2 \\
\hline Risco Nutricional & 2 & 2,1 \\
\hline Desnutrição Leve & 15 & 15,7 \\
\hline Desnutrição Moderada & 1 & 1,0 \\
\hline Desnutrição Grave & 1 & 1,0 \\
\hline
\end{tabular}

Fonte: Dados de pesquisa realizada pelos autores.

Tabela 2 - Distribuição das crianças conforme a situação nutricional (favorável ou desfavorável) e o efeito bruto das variáveis candidíase perineal, candidíase oral e dermatite irritativa das fraldas. Rio Grande-RS, 2008.

\begin{tabular}{|c|c|c|}
\hline \multirow{2}{*}{ Variável } & \multicolumn{2}{|c|}{ Situação Nutricional } \\
\hline & Favorável & Desfavorável \\
\hline Candidíase perineal* & $\mathrm{n}=77$ & $\mathrm{n}=19$ \\
\hline Ausente & $63(81,8 \%)$ & $16(84,2 \%)$ \\
\hline Presente & $14(18,2 \%)$ & $3(15,8 \%)$ \\
\hline \multicolumn{3}{|l|}{ Candidíase oral** } \\
\hline Ausente & $69(89,6 \%)$ & $17(89,5 \%)$ \\
\hline Presente & $8(10,4 \%)$ & $2(10,5 \%)$ \\
\hline \multicolumn{3}{|c|}{ Dermatite Irritativa das fraldas $* * *$} \\
\hline Ausente & $55(71,4 \%)$ & $8(42,1 \%)$ \\
\hline Presente & $22(28,6 \%)$ & $11(57,9 \%)$ \\
\hline
\end{tabular}

$*(p=0,80$ e Fischer $=1) * *(p=0,98$ e Fischer $=1) * * *(p=0,01$ e Fischer $=0,02)$.

Fonte: Dados de pesquisa realizada pelos autores. 


\section{DISCUSSÃO}

No presente estudo, $53 \%$ das crianças atendidas nas consultas de enfermagem em puericultura eram do sexo feminino. Em pesquisa realizada em João Pessoa-PB, sobre o acompanhamento de crianças menores de um ano através de consultas de Puericultura, foi registrado um número maior de crianças do sexo masculino $(71,8 \%)^{(7)}$.

Em relação ao estado nutricional das crianças na última consulta realizada, a maioria (80,2 \%) encontrava-se eutrófica, $2,1 \%$ encontrava-se em risco nutricional e percentual relativamente alto, 17,7\%, apresentavam algum grau de desnutrição. Estudo realizado em zona da mata do Nordeste brasileiro, região com altos índices de desnutrição infantil, encontrou um percentual semelhante de eutróficas $(86,6 \%)$ e um menor de crianças com algum grau de desnutrição $(2,4 \%)^{(8)}$.

Estudos apontam tendência de diminuição da prevalência da desnutrição infantil no Brasil, associada a melhorias de condições sociais, educativas e econômicas, que vêm ocorrendo em especial nas grandes metrópoles. Porém, este problema ainda representa um desafio para diversas regiões, persistindo entre crianças oriundas de áreas socialmente mais vulneráveis, mesmo frente ao maior acesso aos alimentos em nível familiar ${ }^{(9)}$. Além da carência causada no organismo, a desnutrição está associada à incidência e severidade de agravos de saúde e à mortalidade infantil(10).

Os fatores socioambientais devem ser abordados na consulta de enfermagem em Puericultura. Quando os questionamentos sobre o desenvolvimento da criança, o estilo e as condições de vida, problemas psicológicos e o ambiente familiar fazem parte da prática do profissional, desenvolve-se a base da promoção à saúde e pode-se melhorar a relação com a família(4).

No que diz respeito ao encaminhamento para o pediatra, nos casos em que foi realizado, a maioria, 70,65\%, se deu em função de alteração no exame físico. O enfermeiro, após a execução do processo de enfermagem, encaminha as crianças com alterações clínicas que exijam uma avaliação desse profissional. A qualidade dos serviços de saúde está relacionada ao acesso às diversas especialidades, por parte da população (multidisciplinaridade do cuidado) ${ }^{(7)}$.

A prescrição de vitamina A e ferro foi o motivo de 19,56\% dos encaminhamentos para o pediatra. Embora não haja dados nacionais acerca da magnitude do problema de deficiência de vitamina A no Brasil, estudos isolados demonstram que há prevalência de mais de $10 \%$ de crianças com hipovitaminose $\mathrm{A}$, condição caracterizada como problema de saúde pública, segundo a Organização Mundial da Saúde (OMS). O Ministério da Saúde desenvolve também o Programa Nacional de Suplementação de Ferro, que se destina à suplementação preventiva, com sulfato ferroso, de todas as crianças entre 6 a 18 meses $^{(11)}$.

O encaminhamento ao médico para prescrição de vitamina $A$ e ferro remete ao fato de que, no município onde foi realizado o estudo, não há protocolo relativo à assistência em puericultura que permita ao enfermeiro prescrever esses medicamentos de rotina. $\mathrm{O}$ pedido das mães para que as crianças fossem encaminhadas ao pediatra, correspondente ao percentual de $9 \%$, pode demonstrar o quanto elas ainda estão voltadas para o modelo de atenção tradicional, centrado na figura do médico e, muitas vezes, ficam inseguras com a consulta de enfermagem, em especial, quando é uma mãe nova no serviço, aquela que está vindo pela primeira vez ${ }^{(12)}$.

Entre os cuidados essenciais à saúde da criança está o aleitamento materno exclusivo (AME), o qual ocorreu até no máximo o sétimo mês de vida de algumas crianças acompanhadas, apresentando percentual maior o encerramento do AME no terceiro mês $(38,46 \%)$ e menor no sétimo $(3,12 \%)$. A prevalência do AME em menores de 6 meses, em Porto Alegre-RS, em período semelhante ao do presente estudo, foi de $38,2 \%{ }^{(13)}$. Estudo realizado em Teresina-PI constatou que, embora a totalidade de mães pesquisadas reconhecesse a importância do leite materno, mais da metade delas amamentaram exclusivamente seus filhos no máximo por três meses e, ao contrário do esperado, o acompanhamento do bebê e/ou da mãe pela Equipe de Saúde da Família não influenciou no tempo de amamentação(14). A OMS e o Ministério da Saúde recomendam aleitamento materno exclusivo por seis meses e complementado até os dois anos ou mais ${ }^{(11)}$.

O fato de o aleitamento materno exclusivo ter sido mantido até o sétimo mês de vida, mesmo que para uma pequena porcentagem de crianças, pode demonstrar que o serviço contribuiu de forma positiva no incentivo dessa prática. Ressalta-se que a mudança de hábitos, muitos deles fortemente influenciados pela cultura, não é nada fácil e, em alguns casos, apesar de inúmeras tentativas e das diversas estratégias educativas utilizadas, muitas mães abandonam o aleitamento materno logo nos primeiros meses de vida do bebê. Existem mitos e tabus que envolvem a amamentação, o que contribui para sua interrupção precoce ${ }^{(14)}$.

A porcentagem de crianças que abandonou o acompanhamento é elevada (47\%), fato que se pode atribuir à dificuldade de locomoção (seja por condições financeiras ou de acessibilidade) da população até a UBS que, comumente, abrange uma ampla área territorial. Também não há na UBS agentes comunitários de saúde que possam realizar a busca das crianças que abandonaram o acompanhamento.

Estudo realizado em São Leopoldo-RS mostrou que mais de $50 \%$ das crianças não foram acompanhadas de forma regular no primeiro ano de vida nas unidades de atenção primária de saúde, e constatou ainda que as mães procuram atendimento para seus filhos somente quando eles estão doentes. Nesse mesmo estudo a dificuldade de acesso foi o segundo motivo, em frequência, relatado pelos responsáveis para justificarem que seus filhos não foram levados para acompanhamento no serviço de Puericultura ${ }^{(15)}$. Estudo realizado na Paraíba referiu que, no atendimento às crianças, foi comum a ausência de relações de interação, coparticipação e produção de subjetividade entre as mães e o enfermeiro durante a consulta de Puericultura, o que pode favorecer o abandono do acompanhamento $^{(16)}$.

Houve baixa incidência de candidíase oral e perineal, o que pode significar a adesão a orientações preventivas, realizadas durante a consulta de enfermagem. Orientações quanto a medidas de higiene são eficazes para prevenir a candidíase 
oral e perineal na criança e devem fazer parte da consulta de enfermagem em puericultura.

Estudo refere que com a desnutrição aumenta em 4,5 vezes o risco de colonização bucal por Candida albicans, o que pode aumentar o risco de desenvolver candidíase oral ${ }^{(17)}$. A presença de candidíase oral na criança também foi associada à presença de candidíase perineal ${ }^{(18)}$. No presente estudo não houve associação entre candidíase oral e perineal e situação nutricional desfavorável.

A dermatite irritativa de fraldas esteve presente em 34,4\% das crianças. Em estudo realizado no Reino Unido, 16\% das crianças apresentavam dermatite irritativa das fraldas ${ }^{(18)}$. Na consulta de enfermagem em puericultura o enfermeiro deve fornecer orientações com o objetivo de prevenir a ocorrência da dermatite irritativa das fraldas. Essas orientações constituem-se num conjunto de medidas cujos principais objetivos são manter a área das fraldas limpa e seca, limitar a mistura e dispersão da urina e das fezes e reduzir contato das mesmas com a pele da criança, evitar irritação e maceração da pele e preservar a função de barreira cutânea(19).

Quando a criança que vem à consulta de enfermagem em Puericultura já apresenta a dermatite, orientações quanto a medidas de higiene, cuidados e fraldas adequadas são indispensáveis. O acompanhamento das crianças com dermatite irritativa das fraldas deve ser regular, e atenção especial necessita ser dispensada nos períodos diarreicos ou de uso de antibiótico sistêmico. Na criança com dermatite irritativa das fraldas pode ocorrer a infecção secundária por Candida albicans que leva à candidíase perineal, uma das principais complicações dessa dermatite ${ }^{(19)}$.

Houve associação entre dermatite irritativa de fraldas e situação nutricional desfavorável, o que pode apontar uma provável deficiência nas orientações prestadas, bem como a falta de adesão às mesmas. Estudo indica que pouco mais de um terço das mães não adere às orientações dadas durante a consulta de enfermagem, sendo que a maioria justifica como motivo principal para tanto a falta de recursos financeiros ${ }^{(20)}$. É possível entender que os dois fatores associados possam manter relação com a condição econômica; entretanto, para sustentar tal afirmativa, seria necessário o aprofundamento do tópico, por meio de estudos subsequentes.

\section{CONSIDERAÇÕES FINAIS}

A maioria das crianças acompanhadas era do sexo feminino e encontrava-se eutrófica; todas as que necessitaram de avaliação pediátrica foram encaminhadas; a dermatite irritativa das fraldas esteve associada à situação nutricional desfavorável e o aleitamento materno foi realizado, no máximo, até o sétimo mês de vida do bebê.

Como uma limitação do estudo está o fato de não ter envolvido determinantes sociais, como renda e escolaridade materna, as quais podem influenciar na adesão às consultas de enfermagem em puericultura, bem como estarem associadas à situação nutricional desfavorável da criança.

O estudo aponta a necessidade de sensibilizar a população a respeito da importância da puericultura para a prevenção de agravos e promoção da saúde das crianças e ainda para o fato de que é preciso dar condições de acesso ao serviço para a população desfavorecida socioeconomicamente.

\section{AGRADECIMENTOS}

À professora Dra. Maria Cristina Flores, por disponibilizar os programas estatísticos EP-INFO 15.0 e SPSS versão 6.04 e pelos esclarecimentos quanto ao funcionamento dos mesmos.

\section{REFERÊNCIAS}

1. Black RE, Allen LH, Bhutta ZA, Caulfield LE, Onis M, Ezzati $M$, et al. Maternal and child undernutrition: global and regional exposures and health consequenses. Lancet 2008; 371(9608):243-60.

2. Victora CG, Adair L, Fall C, Hallal PC, Martorell R, Richter $\mathrm{L}$, et al. Maternal and child undernutrition: consequences for adult health and human capital. Lancet 2008;371(9609):340-57.

3. Programa das Nações Unidas para o Desenvolvimento [homepage na internet]. Objetivos de Desenvolvimento do Milênio. [acesso em 2 jul 2012]. Disponível em: $<$ www.pnud.org.br/odm $>$.

4. Ministério da Saúde (Brasil). Agenda de Compromissos para a Saúde Integral da Criança e Redução da Mortalidade Infantil. Brasília: Ministério da Saúde; 2005. (Série A - Normas e Manuais Técnicos).

5. Organização Pan-americana da Saúde. Manual do Desenvolvimento Infantil no Contexto do AIDPI. Washington: OPAS; 2005.
6. Falbo BCP, Andrade RD, Furtado MCC, Mello DF. Estímulo ao desenvolvimento infantil: produção do conhecimento em enfermagem. Rev Bras Enferm 2012;65(1):148-54.

7. Carvalho MF, Lira PIC, Romani SAM, Santos IS, Veras AACA, Filho MB. Acompanhamento do crescimento em crianças menores de um ano: situação nos serviços de saúde em Pernambuco, Brasil. Cad Saúde Pública 2008;24(3):675-85.

8. Oliveira JS, Lira PIC, Maia SR, Sequeira LAS, Amorim RCA, Filho MB. Insegurança alimentar e estado nutricional de crianças de Gameleira, zona da mata do Nordeste brasileiro. Rev Bras Saúde Matern Infant 2010;10(2):237-45.

9. Monteiro CA, Benicio MHD, Konno SC, Silva ACF, Lima ALL, Conde WL. Causas do declínio da desnutrição infantil no Brasil, 1996-2007. Rev Saúde Pública 2009;43(1):35-43.

10. Murakami GF, Guimarães MJB, Sarinho SW. Desigualdades sociodemográficas e causas de morte em menores de cinco anos no Estado de Pernambuco. Rev Bras Saúde 
Matern Infant 2011;11(2):139-52.

11. Ministério da Saúde (Brasil). Saúde da criança: nutrição infantil, aleitamento materno e alimentação complementar. Brasília: Ministério da Saúde; 2010. (Cadernos de Atenção Básica, n. 23).

12. Campos RMC, Ribeiro CA, Silva CV, Saparolli ECL. Consulta de enfermagem em puericultura: a vivência do enfermeiro na Estratégia de Saúde da Família. Rev Esc Enferm USP 2011;45(3):566-74.

13. Ministério da Saúde (Brasil), Secretaria de Atenção à Saúde. Departamento de Ações Programáticas e Estratégicas. II Pesquisa de Prevalência de Aleitamento Materno nas Capitais Brasileiras e Distrito Federal/Ministério da Saúde, Secretaria de Atenção à Saúde, Departamento de Ações Programáticas e Estratégicas. Brasília: Ministério da Saúde; 2009. (Série C. Projetos, Programas e Relatórios).

14. Araújo OD, Cunha AL, Lustosa LR, Nery IS, Mendonça RCM, Campelo SMA. Aleitamento materno: fatores que levam ao desmame precoce. Rev Bras Enferm 2008;61(4):488-92.

15. Vitolo MR, Gama CM, Campagnolo PDB. Frequência de utilização do serviço público de puericultura e fatores associados. J Pediatr 2010;86(1):80-4.

16. Assis WD, Collet N, Reichert APS, Sá LD. Processo de trabalho da enfermeira que atua em puericultura nas unidades de saúde da família. Rev Bras Enferm 2011;64(1):38-46.

17. Gaitán-Cepeda LA, Sánchez-Vargas LO, Pavia-Ruz N, Muñoz-Hernández R, Villegas-Ham J, Caballos-Salobreña A. Candida bucal en niños mexicanos con $\mathrm{VIH} /$ sida, desnutrición o marginación social. Rev Panam Salud Pública 2012;31(1):48-53.

18. Adalat S; Wall D; Goodyear H. Diaper dermatitis-frequency and contributory factors in hospital attending children. Pediatr Dermatol 2007;24(5):483-8.

19. Fernandes JD, Machado MCR, Oliveira ZNP. Fisiopatologia da dermatite da área das fraldas - Parte I. An Bras Dermatol 2008;83(6):567-71.

20. Boscatto PC. Adesão das mães às condutas de enfermagem na dermatite irritativa de fraldas: estudo exploratório. Online braz J Nurs (Online) [periódico na internet]. 2008 [acesso em 2 set 2008];7(2) Disponível em: < http://www. uff.br/objnursing/index.php/nursing/issue/archive $>$. 